\title{
Research on the Three Dimensional Detection Technology for Magnetic Sheet in Industrial Production
}

\author{
Wenbo Zhang, Chuo Yang, Yongxin Feng* and Deyu Zhang
}

School of Information Science \& Engineering, Shenyang Ligong University, 110159, Liaoning, China

\begin{abstract}
This study proposes a three-dimensional size detection system for inerratic magnetic sheets according to specific size parameter requirements, such as the volume of the industrial micro magnetic sheet. Based on the noise interference generated by the corresponding detection environment of the magnetic sheet, a hybrid filtering method for the inerratic magnetic sheet is also proposed here, which effectively implements denoising filtering. In the image edge detection module, the edge detection operator most suitable for detecting image edge extraction is selected, and in order to realize the inerratic shape detection in Hough transform, the measurement function of size parameters (including the top surface radius, tilt angle of the profile and top surface, profile thickness, and volume) for the micro cylinder magnetic sheet are designed and implemented. Measurement data, gathered through repeated experiments, builds a more effective detection system for magnetic sheet size than traditional methods at the millimeter level.
\end{abstract}

Keywords: Edge detection, Edge extraction, Magnetic sheet, Hough transform, Hybrid filtering, Three-dimensional size detection.

\section{INTRODUCTION}

Alongside the continuous development of intelligentization and informatization technologies, detection precision and efficiency requirements for all types of micro parts have grown increasingly stringent. To this effect, industrial vision detection technology has become a hot research topic $[1,2]$. Detection of industrial parts generally utilizes artificial contact detection, however, these methods introduce significant errors and do not provide complete information. These problems are not only inherent to the detection method itself, but are often attributable to human error, as well.

Currently, the most commonly utilized industrial production size detection technology is binocular stereo vision [3, 4], which essentially mimics the human eye to achieve threedimensional size detection. Though manual inspection methods do typically perform better, they are disadvantaged by complex computation, longer run time [5]. Two-dimensional visual detecting technology $[6,7]$ is also commonly utilized for size detection, but this method only detects size according to two-dimensional parameters, so its application is subject to certain limitations.

In an effort to further improve the efficiency of machine vision size detection, as well as meet detection precision and efficiency standards according to three-dimensional parameters, a novel method of machine vision size detection in industrial vision inspection systems is urgently needed $[8,9]$. *Address correspondence to this author at the School of Information Sci-
ence \& Engineering, Shenyang Ligong University, 110159 , China; Tel: 86-
24-24682228; Fax: 86-24-24682229; E-mail: fengyongxin@263.net

\section{METHOD OF INERRATIC MAGNETIC SHEET THREE-DIMENSIONAL DETECTION}

\subsection{Camera Calibration}

The Tsai two-step method was used in this study to calibrate the cameras $[10,11]$. Standard boards were calibrated on a checkerboard as shown in Fig. (1).

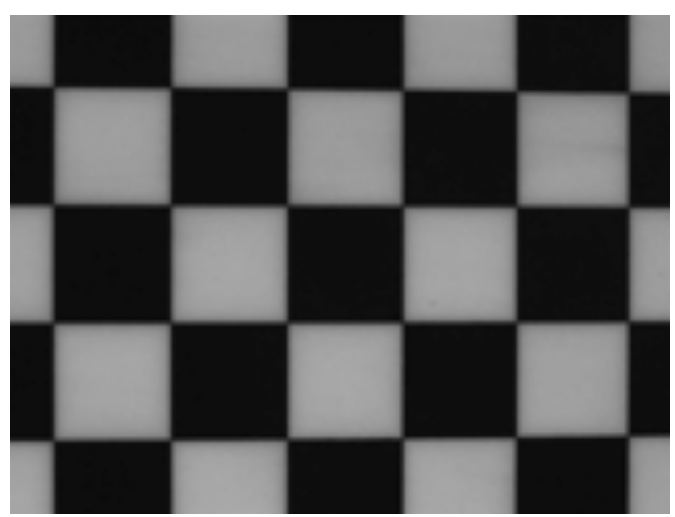

Fig. (1). Calibration checkerboard graphic.

Calibration steps were performed as follows:

(1) Cameras and light source systems were fixed, and the checkerboard calibration board was placed within the scope of the camera's view. The shooting distance was adjusted to ensure that the number of checkerboard points was more than $6 * 4$. The camera was fixed, so its position remained unchangeable.

(2) The world coordinate system of the calibration board was set. The coordinate of the first checkerboard point on the upper left corner of the calibration board 
served as the source point coordinate of the world coordinate system, and the upper vertical area was set as the $\mathrm{Z}$-axis positive direction to dynamically bind the world coordinate system with the pixel coordinate system; in other words, the same results were achieved regardless of checkerboard calibration board movement. Based on the fact that the camera position was unchangeable and that the three-dimensional coordinate point was one of the camera's inputting parameters, camera calibration functioned as static parameter calibration.

(3) The camera was powered on, and dedicated image acquisition software was activated to access images of the calibration board. The images were preprocessed to obtain closer coordinates to the original calibration images.

(4) The Harris corner extraction method of pixel coordinates was utilized to extract the pixel coordinate points corresponding to world coordinate points, forming the initial camera calibration values [12, 13].

(5) Using the world coordinate points obtained from the world coordinate system and the pixel coordinate points obtained from the pixel coordinate system as the inputting initial values, the Tsai two-step calibration procedure was performed to acquire calibration parameters. Calibration results are shown in Table $\mathbf{1 .}$

Table 1. Calibration experiment result.

\begin{tabular}{|c|c|}
\hline Calibration Parameter & Calibration Value \\
\hline \hline Focal distance $\mathrm{f}$ & 7.4456 \\
\hline Center of image(u0, v0) & $(785.835,642.75)$ \\
\hline Distortion coefficient $\mathrm{k}$ & 0.0010 \\
\hline & $-0.9643-0.00200 .5002$ \\
Rotation matrix R & $0.00570 .9996-0.0285$ \\
& -0.26480 .02890 .9639 \\
\hline Translation matrix T & $163.6746 .5263-395.1371$ \\
\hline
\end{tabular}

\subsection{Mixed Filtering Algorithm}

Images are easily affected by environment during the detection process, (by Gaussian noise, impulse noise, and a small amount of relatively weak granular noise). Traditional single filters do not successfully filter images, so this study utilized a mixed filtering method to detect industrial cylinder magnetic sheet images [14]. The pseudo code of this method is as follows:

Import the initial figure AND set the specification of the filtering template

Set the threshold of the grayscale extremum

FOR (the gray value of the first image pixel; the number of all the image pixels; the pixel increment on rows)

\{

IF the gray value is the extremum THEN

IF the extremum $<$ the threshold d THEN

Call the median filtering function
ELSE

Call the Gaussian filtering function

ELSE IF dips or swells by Comparing the gray value with

the surrounding pixels on the template THEN

Call the median filtering function

ELSE

DO NOTHING

\}

The processed results of the cylinder magnetic sheets are shown in Fig. (2). the initial image

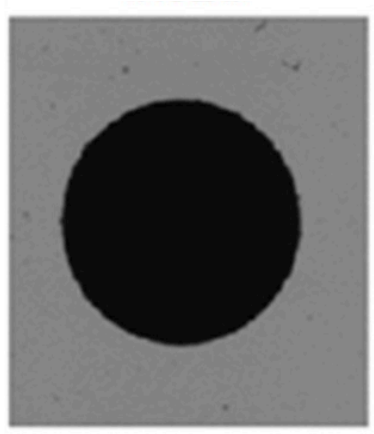

the image after improving filtering

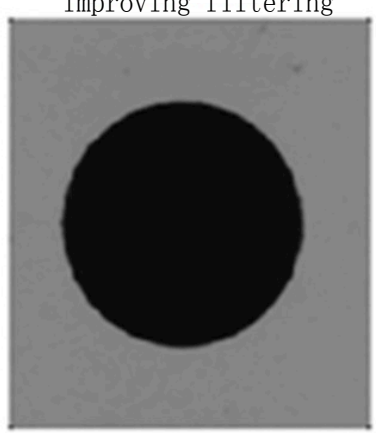

Fig. (2). Filter pre-and-post control graphic.

\subsection{Image Edge Detection and Processing}

Image edge detection and processing is performed to obtain the feature area or feature edges in images under detection. The processing procedure of edge detection takes edge information from the image to be detected and separates it from gray value information in other images, and applies said information to follow-up image analysis and processing. The basic method of traditional edge detection analyzes all pixels in the image to be detected for pixels with gray values different from their surrounding pixels, and analyzes change rules by the first or second derivative of the special pixels to detect image edges.

Commonly accepted rules for edge detection algorithms include the SNR rule, position precision rule, and single edge response rule. These three rules are the main standard for measuring whether an edge detection operator performs successfully or not. After images undergo edge detection in the Sobel operator, the computation of which is relatively simple, the image edges contain complex pocking marks, and lack clarity; compared to other edge detection operators currently in use in the industry, there is a sizeable gap in performance [15]. Images processed in the Canny operator and the Roberts operator are relatively close in quality, however, because the calculation of the Canny operator is highly complex compared to that of the Roberts operator, edge information extracted by the Canny operator results in a small account of loss. To this effect, comprehensively balancing all aspects of all factors, including time and efficiency, the Roberts operator was selected as the edge detection operator in this study [16-18].

The Roberts operator calculates edge detection through a relatively simple process. The primary advantage of the Roberts operator lies in its ability to detect vertical edges in a highly precise manner. In addition to highly precise edge 
position detection, the Roberts operator is sensitive to noise [19]. For images to be detected where edges are more obvious and noise level is relatively low, the Roberts edge detection operator achieves highly effective detection. The corresponding analysis formula is shown below:

$$
G[f(i, j)]=\sqrt{(f[i, j]-f[i+1, j+1])^{2}+(f[i+1, j]-f[i, j+1])^{2}}
$$

In the formula (1), $G[f(i, j)]$ is the gradient amplitude of the edge operator and $f(i, j)$ is the coordinate value of the integer pixel in the target image. The concrete method is to take the differential of the gray value of coordinates, plus or minus 45 pixels, in the images to be detected first, then to perform the sum operation on the results, while simultaneously setting a specific threshold $T$ for the gradient amplitude of each pixel. If $G[f(i, j)] \geq T, f(i, j)$ is the edge point of the result image; if $G[f(i, j)] \leq T, f(i, j)$ is not the edge point, thus the edge detection process is complete. The Roberts edge detection operator, Canny edge detection operator without Gaussian filtering, and Sobel edge detection operator were adopted in turn to extract the feature edges of the same magnetic sheet image. The processing results are shown in Fig. (3).

\subsection{Straight Line Detection and Thickness Measurement}

\subsubsection{Straight Line Detection}

In the rectangular coordinate system of the image space, all lines that pass the point $(x, y)$ can be represented by the following formula:

$$
y=p x+q
$$

In the above formula, $(x, y)$ is a point in the image space, $(p, q)$ is a point in the parameter space, $p$ is the slope, and $q$ is the intercept. Formula (2) can be rewritten as Formula (3) through appropriate deformation:

$q=-p x+y$

Formula (3) is a line which passes the point $(x, y)$ in the parameter space. It is assumed that $\left(x_{i}, y_{i}\right)$ and $\left(x_{j}, y_{j}\right)$ are two different collinear points in the image space, then the following two formulas will be obtained according to Formula (3):

$y_{i}=p x_{i}+q$
$y_{j}=p x_{j}+q$

Points $(p, q)$ were plugged into the following formulas, respectively:

$q=-x_{i} p+y_{i}$

$q=-x_{j} p+y_{j}$

Formulas (6) and (7) express two different lines. It is assumed that they intersect at the points $\left(p^{\prime}, q^{\prime}\right)$, then the points $\left(p^{\prime}, q^{\prime}\right)$ correspond to the line that passes the points $\left(x_{i}, y_{i}\right)$ and the points $\left(x_{j}, y_{j}\right)$ in the image space. Conversely, each point on the line which passes $\left(x_{i}, y_{i}\right)$ and $\left(x_{j}, y_{j}\right)$ in the image space corresponds to a certain line in the parame-

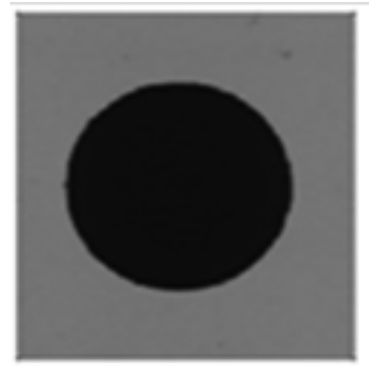

a) the image after denoising

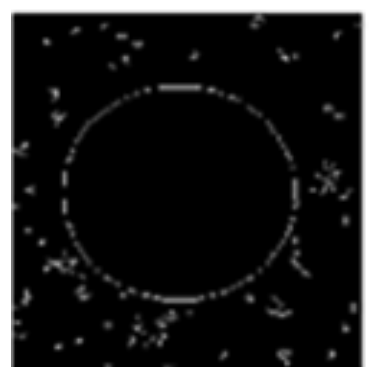

c) sobe 1

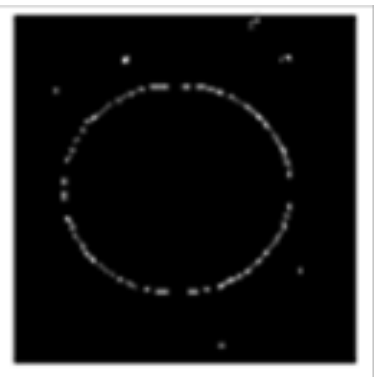

b) canny

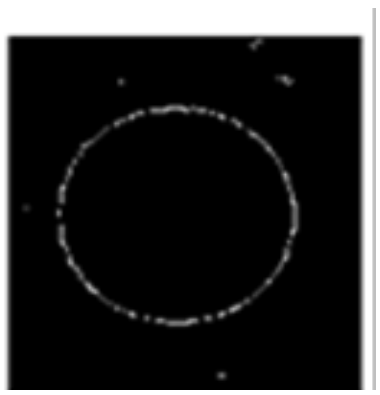

d) roberts

Fig. (3). Edge detection algorithm processing result. 
ter space. These lines intersect at the points $\left(p^{\prime}, q^{\prime}\right)$ in the $(p, q)$ space. Therefore, the lines in the image space to be detected can be transformed to solve the intersection point of multiple lines in the corresponding parameter space.

The dual relationship of the dotted line in the Hough transform reflects the collinear points in the image space as they correspond to the intersected lines in the parameter space, and all lines intersected at any point in the parameter space correspond to the collinear points in the image space $[20,21]$. An instance where the Hough transform points and lines in the image space and the parameter space are in accordance with each other is shown in [22]

When a detected line is vertical, the value of $p$ can be infinity. To remedy this, a following polar coordinate system is typically adopted to express the lines in the parameter space

$\rho=x \cos \theta+y \sin \theta \quad \theta \in[0,180]$

A certain point $(x, y)$ in the image coordinate system is transformed to its corresponding sine curve in the $(\rho, \theta)$ parameter space. At that time, the duality between points and lines in the Hough transform is also altered to form dual transformation between the points and the sine curves.

Under Hough transform, all collinear points in the rectangle coordinate system can be transformed into the cluster of sine curves which intersect at the same point in the polar coordinate system, maintaining one-to-one correspondence as shown in Fig. (4).
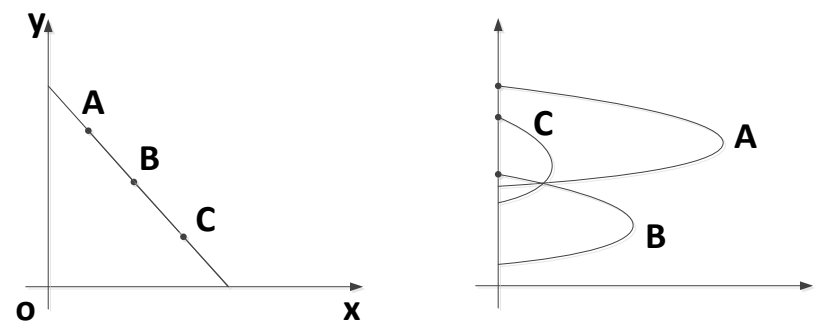

Fig. (4). Relation between rectangular coordinate and polar coordinate.

To detect lines in the image coordinate system, the $\theta$ and $\rho$ in the parameter coordinate space were quantified, as shown in Fig. (4b). According to the coordinate $(x, y)$ of the point in the rectangle coordinate, the value of each $\rho$ is calculated in the range of $\theta$, as shown in Fig. (4a). It is assumed that the calculated value of $\rho$ is equal to the pixel gray value of a quantified grid or within the approximate range, then the accumulator that corresponds to the grid adds 1. After all points in the rectangle coordinate system are passed by Hough transform and the results are counted, the accumulator in each grid is counted and analyzed. If there is only one line in the image to be detected, parameter values $\left(\rho^{*}, \theta^{*}\right)$ in the rectangle coordinate system corresponding to the accumulator form the requested line expression. If there is more than one line in the image to be detected, a corresponding threshold is set for the statistic value of each grid. Only within the scope of the threshold can the value be the count value of the accumulator on the line.

If an array $A(\rho, \theta)$ is used to record the quantified $\theta$ and the corresponding $\rho$, then the line detection based on Hough transform can be described as follows:

1) According to precision requirements, $\rho$ and $\theta$ are quantified within the scope of $\rho$ and $\theta$, which are respectively divided into $m$ and $n$ portions each expressed by $\rho_{i}$ and $\theta_{j}$.

2) The image is traversed from top to bottom and from left to right. If the current detected point $(x, y)$ is the edge target point, each value of $\rho_{i}$ corresponding to $\theta_{j}$ is calculated according to Formula (8).

3) $A$ is accumulated according to $\rho_{i}$ and $\theta_{j}$, that is:

$$
A\left(\theta_{j}, \rho_{i}\right)=A\left(\theta_{j}, \rho_{i}\right)+1
$$

4) The second and third steps are performed repetitively until all target points are processed.

5) The maximum value is found in the parameter plane by comparing the element values in the array. This identifies the corresponding line expressed by Formula (1), as well as the number of collinear points. Thus, the polar coordinate parameters corresponding to the lines are:

$\left[\theta^{*}, \rho^{*}\right]=\arg \max A\left(\theta_{j}, \rho_{i}\right), j \in[0, n], i \in[0, m]$

The final detected collinear formula is:

$\rho^{*}=x \cos \theta^{*}+y \sin \theta^{*}$

If there is more than one line in the detection plane, a threshold can be set. If the value of $A\left(\theta_{j}, \rho_{i}\right)$ is greater than the threshold, the points in the image space corresponding to the curves passing the point $(x, y)$ are considered collinear; conversely, if the value of $A\left(\theta_{j}, \rho_{i}\right)$ is smaller, the points in the image space corresponding to the curves passing the point are considered isolated points.

If the quantified $\theta$ and $\rho$ are too rough, the resultant line parameters are not accurate. Quantification must not introduce too much complexity to calculation, however. Therefore, this study processed the quantification of $\theta$ and $\rho$ under precision requirements while maintaining simplicity.

\subsubsection{Thickness Measurement}

The obtained thickness image of the cylinder magnetic sheet to be detected underwent image preprocessing as shown in Fig. (6). 


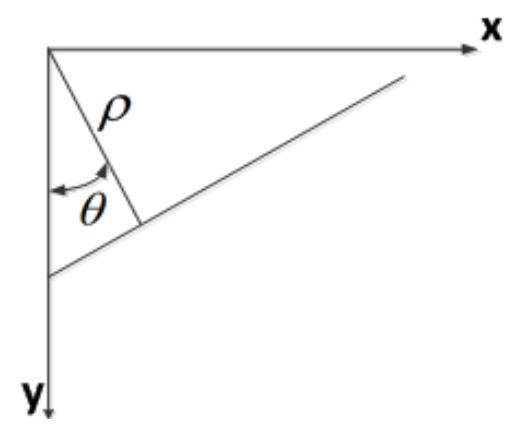

a) Parameter geometric sketch of the line In the polar coordinate expression form

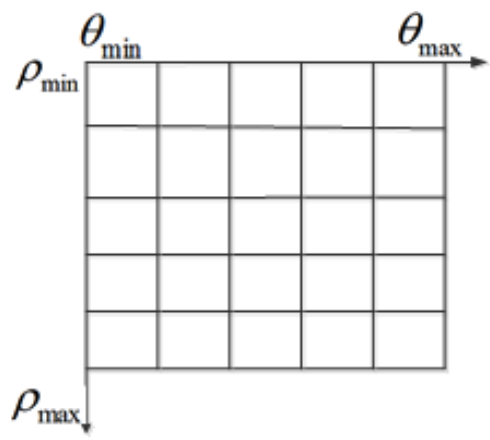

b) Pixel discrete quatization

Fig. (5). Linear detection using Hough transform.

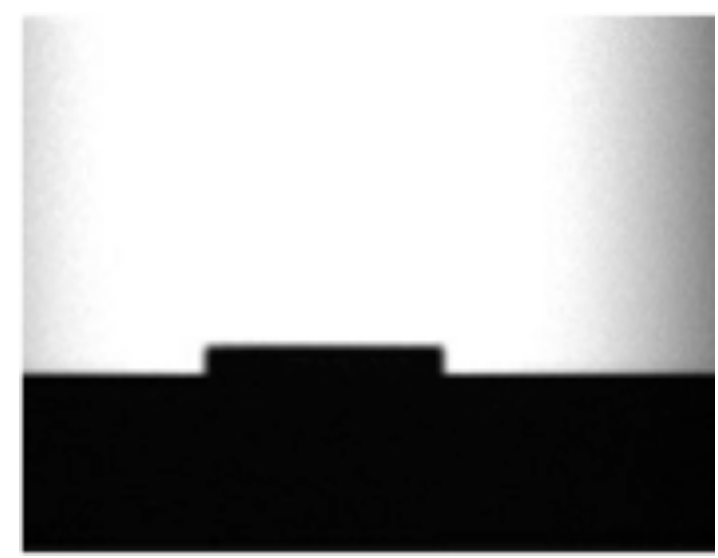

Fig. (6). Magnetic sheet thickness image before detection.

The image edge detection algorithm was applied to preprocessed images to obtain feature edges as shown in Fig. (7). As far as size parameters are concerned, as mentioned above, there are multiple lines in an image which must be detected. This is illustrated in Fig. (8).

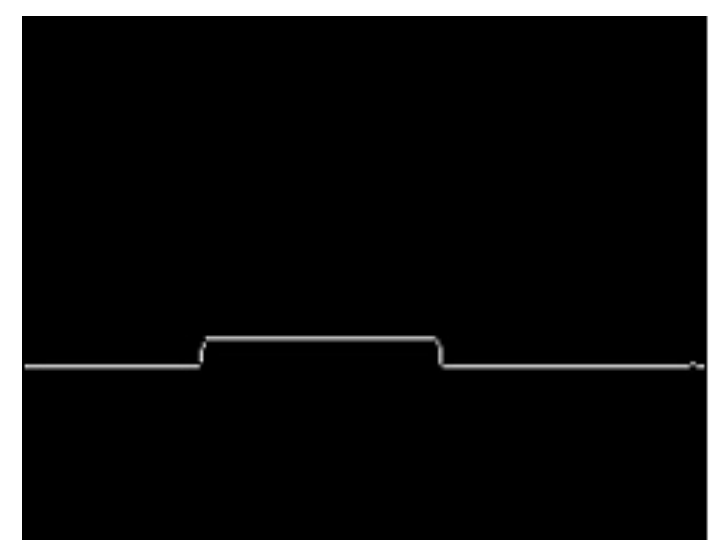

Fig. (7). Magnetic sheet thickness edge image.

The two-dimensional size parameter obtained through the thickness image mainly consists of the thickness of the magnetic sheet side face and the tilt angle of the top and bottom surfaces and the side height. As shown in Fig. (8), the thickness is $d$, and the tilt angle is $a, b$.

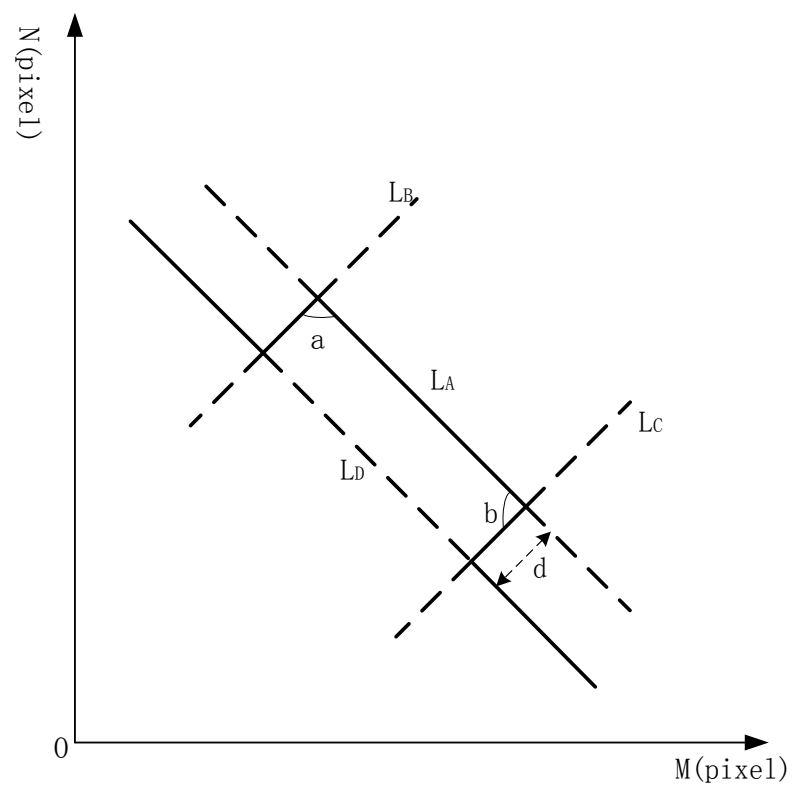

Fig. (8). Thickness coordination analysis image.

The detection and recognition of a straight line is the basis if follow-up detection of the thickness and tilt angle. Because the top surface diameter of the thickness image of a cylindrical magnetic sheet is far larger than the thickness of the thickness image of a cylindrical magnetic sheet, each detected line pair can be distinguished by identifying the distribution of the statistical values of $A\left(\theta_{j}, \rho_{i}\right)$. The statistical results of the $A\left(\theta_{j}, \rho_{i}\right)$ values showed four peaks throughout the line detection process - two higher, and two lower peaks. Clearly, the higher peaks are the two long lines of the image to be detected, (in other words, the diameter of the magnetic sheet projected to the thickness image.) The two lower peaks are the corresponding short lines of the side face thickness. Only when the threshold is set accurately is the peak near the appropriate $A\left(\theta_{j}, \rho_{i}\right)$ value appropriate. Multiple lines in the image can then be distinguished, thus the detection and recognition of the straight line is fully realized. 
After completing line detection and recognition tasks, it is further necessary to measure the profile height tilt angle. The following method was used to measure the angle between the lines.

Because the analytical expression of each line is obtained by the Hough transform of the polar coordinate, one of the necessary parameters for angle measurement is the tilt angle $\theta$ of the line. To obtain the angle between the line $L_{A}$ and the line $L_{B}$, the parameters in the expression of the two lines can be directly subtracted to identify absolute value; thus identifying the angle between $L_{A}$ and $L_{C}$. In this study, deviation in the parallelism of the cylindrical magnetic sheet top and bottom surfaces is negligible. As shown in Fig. (8), the angle between $L_{A}$ and $L_{B}$ is equal to the angle between $L_{B}$ and $L_{D}$; in the same method, the angle between $L_{A}$ and $L_{C}$ is equal to the angle between $L_{C}$ and $L_{D}$.

Certain permanent interference occurs when images to be detected are taken at the corner between the lines, causing vagueness at the corners. Interference is not caused by issues with quality of the magnetic sheet. Because measuring the thickness $d$ directly readily results in error, it is not applicable to high-precision size measurement of two-dimensional size. To measure thickness at sufficiently high precision, a novel method for measuring the thickness of circular magnetic sheets is necessary.

This study obtained thickness parameters by measuring the distance between parallel lines to indirectly describe thickness; line $a$ and line $b$ are the opposite side lines and the values of parameters $\theta$ and $\rho$ are known. One of these is selected as the benchmark (here the line $a$ was selected), then the edge images are scanned individually by row and column, and each target pixel is calculated to determine whether or not it is on the line $b$. If the pixel is on the line $b$ , the distance between the pixel and the line $a$ is calculated, effectively calculating all the vertical distances between all target pixels on the line $b$ and the line $a$. At the end of this process, the average value is the distance between the corresponding lines of line $a$ and line $b$.

\subsection{Circle Detection and Feature Size Measurement}

\subsubsection{Circle Detection}

For a circle with radius $r$ and center $(a, b)$, the equation of the rectangle coordinate system can be expressed as follows:

$(x-a)^{2}+(y-b)^{2}=r^{2}$

The expression of the polar coordinate is:

$x=x_{0}+r \cos \theta$

$y=y_{0}+r \sin \theta$

So the parameter formula of the circle in the parameter space $O-a b$ is:

$(a-x)^{2}+(b-y)^{2}=r^{2}$
The expression of the polar coordinate is:

$a=x-r \cos \theta$

$b=y-r \sin \theta$

A circle in the parameter space $\mathrm{O}-\mathrm{ab}$ corresponds to a point $(x, y)$ in the $\mathrm{O}$-xy space. All concyclic points in the $\mathrm{O}$ xy space and the corresponding circles in the O-ab space intersect at a point - this point is the center coordinate of the concyclic points in the $\mathrm{O}$-xy space.

The above analysis demonstrates that in order to solve all three parameters of the circle equation, a three-dimensional grid and three-dimensional array must be established for a point in the O-xy space corresponding to a three-dimensional quadric surface in the $\mathrm{O}-\mathrm{ab}$ space. If the surface passes a specific grid, the value of the corresponding threedimensional calculation unit will add 1 . The method of detecting concyclic points is consistent with the method of detecting collinear points, basically, but at the expense of increased calculation complexity. Formula (16) was rewritten as follows, in order to allow convenient calculation:

$a=x-r \cos \theta \Rightarrow r=(x-a) / \cos \theta$

Therefore,

$b=y-r \sin \theta$

$\Rightarrow b=y-(x-a) \sin \theta / \cos \theta$

$\Rightarrow b=a \tan \theta-x \tan \theta+y$

In the formulas (18) and (19), $\theta$ is the gradient angle of the current point. The mapping of the circle in the parameter space can be obtained through Formula (19), and the parameters drop from three to two simultaneously.

According to the above principles, the concrete steps of the circle detection algorithm based on Hough transform are as follows:

1) According to precision requirements, $a$ and $b$ are quantified within the scope of $a$ and $b$, which are respectively divided into $m$ and $n$ portions each of which is expressed by $a_{j}$ and $b_{i}$.

2) The gradient, gradient direction of the original image, and tangent value are calculated.

3) The image is traversed from top to bottom and from left to right. If the current detected point $(x, y)$ is the edge target point, the gradient tangent value corresponding to the current point is searched and each value of $b_{i}$ that $a_{j}$ corresponds to is calculated according to Formula (19).

4) $\quad A$ is accumulated according to $a_{j}$ and $b_{i}$, that is:

$$
A\left(a_{j}, b_{i}\right)=A\left(a_{j}, b_{i}\right)+1
$$


5) According to the lines obtained through Formula (19) in the parameter space, the count of the point on the line adds 1 .

6) The third and fifth steps are performed repetitively until all target points are processed.

7) The point of maximum count in the parameter space (the center expressed by Formulas (4-11), in other words,) is obtained.

8) The coordinate of the circle center is defined and plugged into Formula (12) or the circle in the image space to obtain the radius of the circle.

\subsubsection{Radius Measurement}

An image of the cylinder top surface detected in our experiment is shown in Fig. (9a). The camera took the images and transmitted them to the computer, then the edge profile images were obtained through image preprocessing as shown in Fig. (9b). Circular detection processing for the edge profile images was then performed to obtain the radius and center of the circular part.

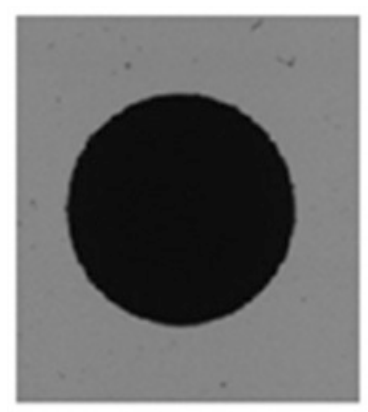

a) the image after denoising

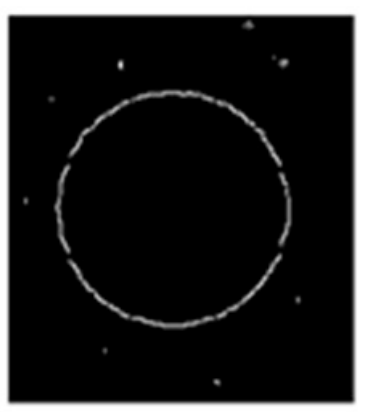

b) the edge detection
Fig. (9). Magnetic sheet upper surface image.

According to the thickness and radius defined by Hough transform, the miniature cylinder volume parameters can be obtained by the volume formula, $V=h \pi r^{2}$.

\section{EXPERIMENT RESULTS AND DATA ANALYSIS}

This system provided size measurement information for small cylinder magnetic sheets sized $2.5 \mathrm{~mm} * 0.5 \mathrm{~mm}$. The theoretical value of the top surface radius is $1.25 \mathrm{~mm}$, the theoretical value of the side thickness is $0.5 \mathrm{~mm}$, the theoretical value of the tilt angle of the side height is 90 , and the theoretical value of the volume of the magnetic sheet is $2.4543 \mathrm{~mm}^{3}$.

To confirm that the proposed detection system meets basic functional industrial detection requirements, as well as size measurement precision requirements for miniature cylinder magnetic sheets, the stability of the system's detection accuracy was also carefully analyzed. In this study, the standard cylinder magnetic sheet was detected 10 times repeatedly, and the average error and the standard deviation of the measured values were used to weigh the stability and detection precision of the results. Formula (21) is the calculation formula for average error and standard deviation. $\tilde{x}$ was the average error, $\hat{x}$ is the standard value to be detected, $x_{i}$ is the value measured for each iteration, $\bar{x}$ is the measured average value, and $n$ is the measured number of iterations. Experiment data is presented in Table 2.

$\tilde{x}=\frac{1}{n} \sum_{i=1}^{n}\left|x_{i}-\hat{x}\right| \quad \sigma=\sqrt{\frac{1}{n} \sum_{i=1}^{n}\left(x_{i}-\bar{x}\right)^{2}}$

All the results of the repetitive experiment are shown in Table 2, including the height left tilt angle, height right tilt angle, top surface radius, volume of the magnetic sheet, and running time of the entire detection system. Corresponding average error and standard deviation were also calculated according to the obtained data to analyze the accuracy and stability of the detection system.

Tilt angle information was included in effort to minimize (or eliminate) error in volume detection that results from

Table 2. Cylinder magnetic sheet measurement result.

\begin{tabular}{|c|c|c|c|c|c|c|}
\hline $\begin{array}{c}\text { Number of } \\
\text { Detected Parts }\end{array}$ & $\begin{array}{c}\text { height Left } \\
\text { Tilt Angle ( }{ }^{\circ} \text { ) }\end{array}$ & $\begin{array}{l}\text { Height Right } \\
\text { Tilt Angle ( }{ }^{\circ} \text { ) }\end{array}$ & $\begin{array}{l}\text { Top Surface } \\
\text { Radius (mm) }\end{array}$ & $\begin{array}{c}\text { Profile } \\
\text { Thickness (mm) }\end{array}$ & $\begin{array}{l}\text { Magnetic Sheet } \\
\text { Volume }\left(\mathbf{m m}^{3}\right)\end{array}$ & $\begin{array}{l}\text { Detection } \\
\text { Time (ms) }\end{array}$ \\
\hline 1 & 90.023 & 89.974 & 1.24997 & 0.50102 & 2.4591 & 40.986 \\
\hline 2 & 90.018 & 89.981 & 1.24985 & 0.50174 & 2.4623 & 40.966 \\
\hline 3 & 90.089 & 89.975 & 1.25101 & 0.50168 & 2.4666 & 41.089 \\
\hline 6 & 90.021 & 90.014 & 1.24973 & 0.49802 & 2.4436 & 40.998 \\
\hline 7 & 89.992 & 89.946 & 1.25082 & 0.50116 & 2.4633 & 40.923 \\
\hline 8 & 90.026 & 89.988 & 1.25009 & 0.50222 & 2.4656 & 41.035 \\
\hline standard deviation & 0.0186637 & 0.0172506 & 0.00059 & 0.001759 & 0.00804 & 0.05348 \\
\hline
\end{tabular}



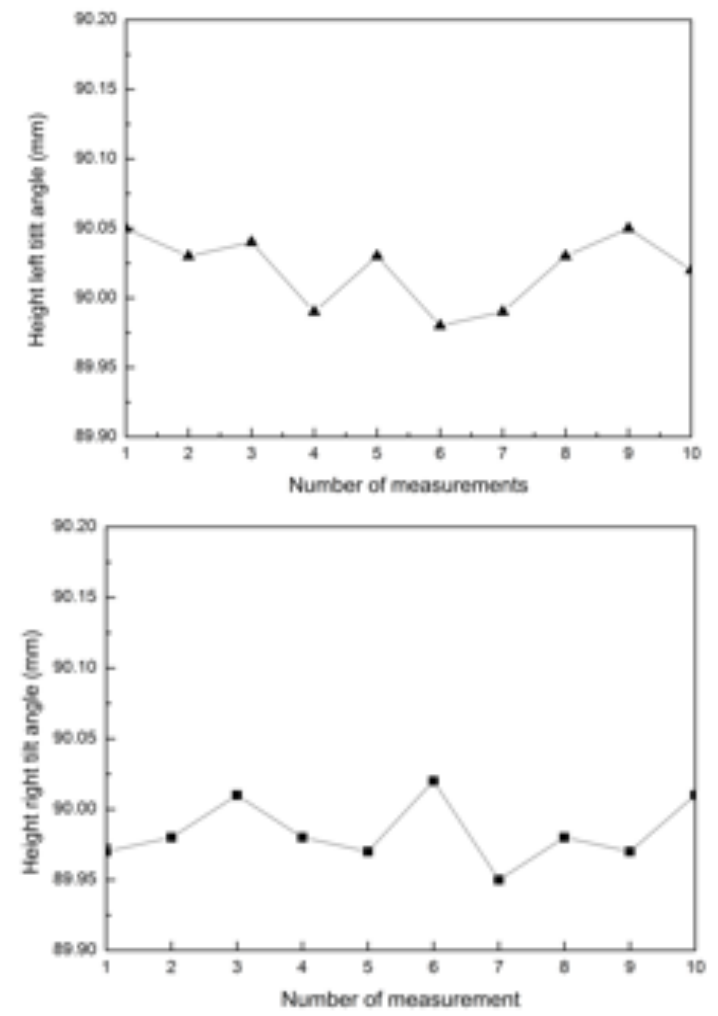

Fig. (10). Height angle measurement value distribution.
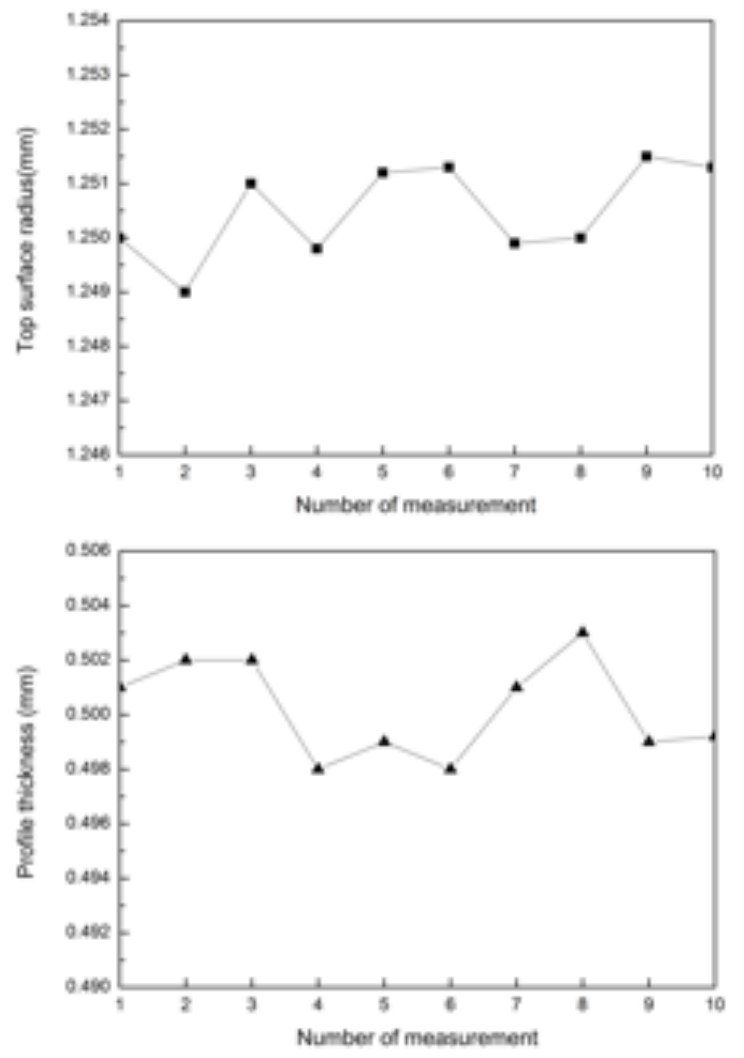

Fig. (11). Upper surface radius and side thickness measurement value distribution.

distorted magnetic sheets. In Fig. (10), the left side shows the distribution diagram of the left tilt angle detection results, with average error of $0.017^{\circ}$, the right side's average error is $0.022^{\circ}$, and both average values are close to the theoretical values. The standard deviation confirms that the real value fluctuates above and below the standard value of the tilt angle. Average detection precision is about $0.02^{\circ}$, suggesting favorable detection stability in the proposed system.
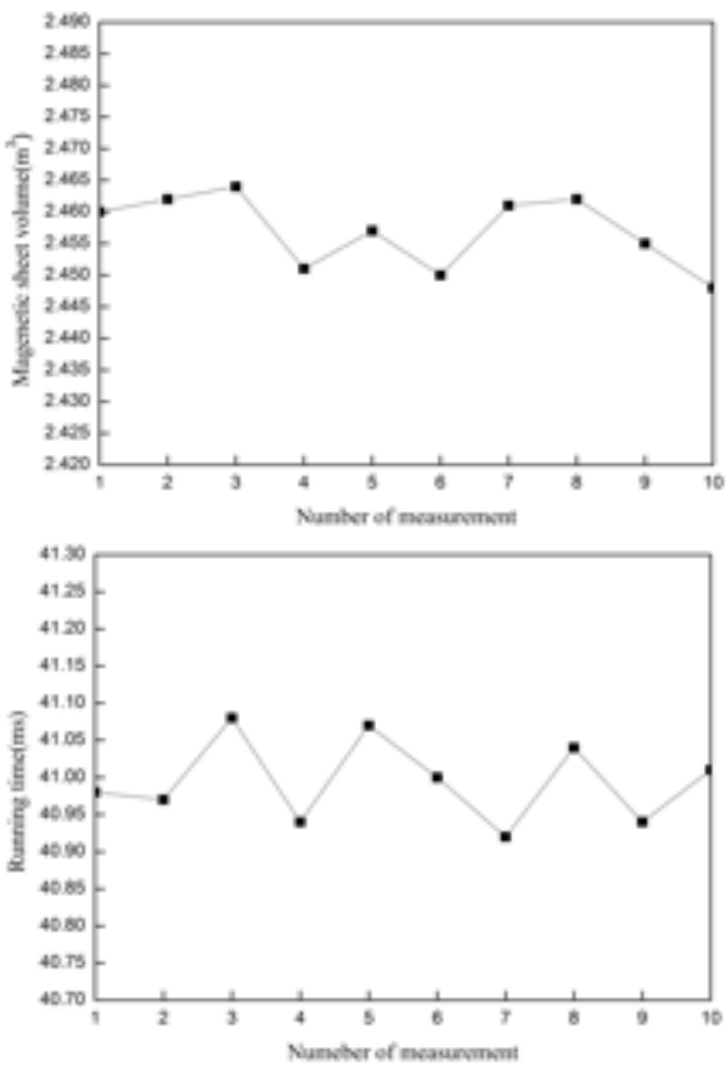

Fig. (12). Magnetic sheet volume result value and processing time distribution.

All two-dimensional parameters of the size detection system in this study (the top surface radius and the thickness of the side) are described in Fig. (11). A distribution diagram of the detection results of the magnetic sheet top surface radius is on the left, with average error of $0.00067 \mathrm{~mm}$, and on the right is the detection results of the corresponding diagram, with average error of $0.01734 \mathrm{~mm}$; the average error of two-dimensional size detection is $0.00702 \mathrm{~mm}$. Standard deviation confirms that the resultant values of the top surface radius and thickness of the side fluctuate within a small range, and that the repetitive detection efficiency of the twodimensional size parameters is effective.

Fig. (12) shows the data distribution diagram of the results for the entire running time of the system, including volume and size parameters of the cylinder magnetic sheet. The precision of the magnetic sheet volume detection was $0.007 \mathrm{~mm}^{3}$. The above table shows that whenever the corresponding standard deviation of parameters to be detected was smaller, the scope of detection values was reasonable and lacking any obvious fluctuation. In other words, the proposed detection system runs smoothly with high repeatability and stability. 


\section{CONCLUSION}

Notably, the run time of the detection system proposed in this study is better than that of the traditional binocular stereo vision detecting system. The run time of just volume parameter detection for the inerratic cylinder magnetic sheet was around $41 \mathrm{~ms}$. To research three-dimensional volume detection on a magnetic sheet with the same specification, binocular stereo vision detecting technology usually requires hundreds of milliseconds. The method proposed in this study retains the detection precision of three-dimensional size parameters while still allowing efficient run time.

That said, due to limited time and the experimental conditions, further research on precision and detection speed is required in order to ensure that the inerratic cylinder magnetic sheet detection technology proposed in this study meets industrial production needs in practice.

\section{CONFLICT OF INTEREST}

The authors confirm that this article content has no conflict of interest.

\section{ACKNOWLEDGEMENTS}

This paper is sponsored by the New Century Program for Excellent Talents of the Ministry of Education of China, Liaoning province innovation group project (LT2011005), the Shenyang Ligong University Computer Science and Technology Key Discipline Open Foundation (2012,2013), Liaoning fourth batch of distinguished professor project (2014) and Liaoning BaiQianWan Talents Program (2014921042).

\section{REFERENCES}

[1] D. F. Wu, "Principle and Application Research of Vision Detection Technology Based On Computer," no. 11, pp. 153-154, 2014.

[2] Q. J. Tang, X. B. Tian, M. C. Geng, Y. Zhou, and T. S. Zhao, "Global calibration method for CCD based visual inspective system," Optics and Precision Engineering, vol. 19, no. 8, pp. 1903-1910, 2011.

[3] J. W. Liu, J. Liang, X. H. Liang, J. M. Cao, and D. Zhang, "Industrial vision measuring system for large dimension work-pieces," Optical Precision Engineering, vol. 18, no. 1, pp. 126-134, 2010.

[4] H. J. Cheng, "The Analysis of the Technology and the Future Application of the Binocular Stereo Vision System," Computer Knowledge and Technology, vol. 7, no. 9, pp. 2145-2147, 2011.
[5] Q. Shang, Q. Q. Ruan, and X. L. Li, "Target Recognition and Location Based on Binocular Stereo Vision," Journal of intelligent systems, vol. 11, no. 4, pp. 303-311, 2011.

[6] W. B. Li, C. H. Lu, J. Li, and J. C. Zhang, "Development and Perspective of Vision Inspection Technology for Surface Defect of Steel Bar," Nondestructive Testing, vol. 34, no. 5, pp. 54-58, 2012.

[7] X. L. Zhu, C. H. Gao, B. W. He, and M. C. Huang, "Investigation into vision detection system for 2D geometric features of mechanical parts," Chinese Journal of Construction Machinery, vol. 8, no. 2, pp. 199-203, 2010.

[8] J. Zhou, K. Ren, Y. Q. Shuai, and Y. H. Chen, "Machine Vision Based Defect Detection On Magnetic Steel Sheet," Journal of Mechanical \& Electrical Engineering, vol. 31, no. 12, pp. 1542-1546, 2014.

[9] G. L. Yan, J. Fu, and Y. N. Yang, "Parts size detection technology based on machine vision," Technology Innovation and Application, vol. 32, pp. 112, 2013.

[10] J. Xu, "Analyzing and Improving the Tsai Camera Calibration Method in Machine Vision," Computer Engineering \& Science, vol. 32, no. 4, pp. 45-48, 2010.

[11] J. Wu, G. Xu, Z. L. Dong, and J. J. Wang, "An Improved Tsai's Twostage Camera Calibration Approach Using Vanish Point Constrain," Geomatics and Information Science of Wuhan University, vol. 37, no. 1, pp. 18-21, 2012.

[12] Q. J. Gao, P. Xu, and L. Yang, "Breakage detection for grid images based on improved Harris corner," Journal of Computer Engineering, vol. 32, no. 3, pp. 766-769, 2012.

[13] C. P. Zhang, and X. G. Wei, "Rectangle detection based on Harris corner," Optics and Precision Engineering, vol. 22, no. 8, pp. 22602266, 2014.

[14] D. G. Wu, and L. P. Zhao, "A Filtering Algorithm for Removing Image Mixed Noise," Process Automation Instrumentation, vol. 33, no. 9, pp. 11-13, 2012.

[15] J. J. Zhang, and J. Luo, "A surface crack edge detection algorithm based on improved Sobeloperator," Journal of Hefei University of Technology (Natural Science), vol. 34, no. 6, pp. 845-847, 2011.

[16] Z. W. Wang, "Comparison research of capability of several detection operators for edge detection," Manufacturing Automation, vol. 34, no. 6, pp. 14-16, 2012.

[17] J. Wang, Z. L. Li, D. X. He, B. and Wang, Z. Li, "Several Operators Based On Edge Detection and Its Features," Journal of Yanan University (Natural Science Edition), vol. 2014, no. 01, pp. 1-8, 2014.

[18] F. H. Huang, Q. F. Liu, and J. F. Ji, "Operators for Edge Detection in Digital Image Based on MATLAB," Mechanical Engineering \& Automation, no. 4, pp. 48-50, 2011.

[19] W. Feng, J. L. Zhang, and Q. J. Miao, "Evaluation of several typical edge detection operator," Electronic Design Engineering, vol. 19, no. 4, pp. 131-133, 2011.

[20] N. Dai, "Based on Hough transform the research and parameter analysis of circle detection algorithm," China metrology, vol. 2010, no. 4, pp. 87-89, 2010.

[21] R. J. Duan, W. Zhao, S. L. Huang, and J. Y. Chen, "Fast Line Detection Algorithm Based On Improved Hough Transform Action," Chinese Journal of Scientific Instrument, vol. 31, no. 12, pp. 2774-2780, 2010.

[22] T. Wada, T. Fujii, and T. Matsuyama, "The $\gamma-\omega$ Hough Transform: Linearizing Voting Curves in an Unbiased $\rho-\theta$ Parameter Space," Systems and Computers in Japan, vol. 24, no. 6, pp. 1-20, 1993. 